\title{
AS AULAS DE EDUCAÇÃO FÍSICA NO ENSINO MEDIO: IMPLICAÇÕES NAS DISCUSSÕES SOBRE AS TEMÁTICAS APRENDIZAGEM E QUALIDADE DE VIDA
}

Rodrigo Castelo Branco de Andrade

Joaquim Antunes Neto

\section{Resumo}

O conceito de qualidade de vida engloba vários aspectos como social, afetivo, econômico, entre outros, por se tratar de VIDA, porém o foco será sobre o aspecto SAÚDE e tentaremos relacioná-lo no interior da educação formal. Dentro do âmbito escolar, sabemos que é na etapa do ensino médio da escolarização que ocorre o aprofundamento de idéias e a reflexão mais completa sobre a ação. Através de um questionário analisamos o índice de massa corporal (do aluno e familiares), questões sobre saúde e atividade física, vendo como foi tratado e como gostariam de ser tratados tais temas durante as aulas de Educação Física. Alunos de uma escola particular do ensino médio (alunos $\mathrm{n}=20$; alunas $\mathrm{n}=15$ ) participaram do estudo. Por meio de tratamento estatístico (teste ANOVA e pós-teste de Tukey), indicamos como valor de significância $\mathrm{p}<0.05$. Os resultados mostraram que a temática foi pouco explorada, e questões sobre obesidade, sedentarismo precoce e doenças crônico-degenerativas poderiam e devem ser tratadas com mais enfoque, visto os resultados e relacionando os mesmos com revisões bibliográficas pertinentes ao estudo.

\section{THE LESSONS OF PHYSICAL EDUCATION IN AVERAGE EDUCATION: IMPLICATIONS IN THE QUARRELS ON THEMATIC THE LEARNING AND QUALITY OF LIFE}

Rodrigo Castelo Branco de Andrade

Joaquim Antunes Neto

\begin{abstract}
The concept of quality of life contains some aspects like social, affective, economical, because we are treating about LIFE, but the focus will be about HEALTH, and we will try relate it inside of formal education, using the Phisical Education as an instrument. Inside the schools we know that is in this part of education (high school) that happens the deepning of ideas and a bigger reflection about the actions. Through a open questionnaire that consisted an index of corporal mass analyses (from students and their parents), we analysed questions about physical activities and heath seeing how this subject has been treating and how they wish that this subject would be treat inside the physical education's classes. Were volunteers a private school's students (boys $=20$ and girls $=15$ ). By statistics treatment ( ANOVA's test and Tukey's post test) we indicate as a significance value $p<0,05$. The results showed that the subject was badly directed, and questions about obesity, precocious sedentarysm and chronicdegenerative deseases could and should be treat with more focus seein the results and relating them with pertinent bibliographical revision.
\end{abstract}




\section{INTRODUÇÃO}

Definir o sentido de algumas palavras-chaves dará subsídios ao leitor para compreender a finalidade deste trabalho, que pretende ter sua relevância no âmbito escolar, uma vez que analisaremos informações referentes a temática atividade física, saúde e qualidade de vida.

Muito se comenta a respeito do conceito e da definição de qualidade de vida, mas pouco se concretiza como verdade. A resposta para essa amplitude de conceitos incertos esta no conflito entre o nível e a qualidade de vida. Muitos consideram o ter, a posse estritamente importante para o alcance de marcas na escala socioeconômica da vida moderna. Isso somente caracteriza o nível em que tal pessoa esta inserida, mas será que a mesma USUFRUI todas essas posses?

Um conceito pertinente de qualidade de vida e aquele que traduz o quanto uma pessoa efetivamente usufrui todas suas posses (estilo de vida), não só materiais, mas também naturais e espirituais. Dentro dos dotes naturais, podemos citar o intelectual e as capacidades físicas inatas. Para o desenvolvimento de tais capacidades, temos que gerar qualquer movimento corporal produzido pelos músculos esqueléticos que resulte em gasto energético maior do que os níveis de repouso, denominado atividade física, segundo Caspersen (1985).

A Organização Mundial da Saúde (The WHOQOL Group, 1995 p. 142) conceitua a qualidade de vida como:

"A percepção do individuo de sua posição na vida no contexto da cultura e sistema de valores nos quais ele vive e em relação aos seus objetivos, expectativas, padrões e preocupações (p. 142).

Aprofundando na questão da saúde, a fisiologia do exercício nos mostra inúmeros estudos sustentando a tese de que existe uma relação entre a pratica de atividade física e uma conduta saudável. Saúde e definida, segundo Bouchard (1990), como condição humana com dimensões física, social e psicológica, cada uma caracterizada por um continuum com pólos positivos e negativos. A saúde positiva estaria associada a capacidade de apreciar a vida e resistir aos desafios do cotidiano e a saúde negativa associar-se-ia a morbidade e, no extremo, a mortalidade.

Porem, sabemos como a visão humanista e enfatizada na disciplina Educação Física. Não cabe criticar tal visão e sim alertar para outras concepções tão importantes quanta o lado humanista. Acreditamos que o 
dilema histórico entre os conteúdos ditos "biologizantes" e "acriticos" e aqueles de valorização de uma "cultura corporal" já esteja superado. A proposta eficaz dos conteúdos da Educação Física será a que valorizar o ser humano em sua totalidade, não podendo, assim, a temática atividade física, saúde e qualidade de vida ser excluída desta nova tendência.

Conhecer as competências da Educação Física no Ensino Médio, tomando como base os Parâmetros Curriculares Nacionais - PCN (1997), permite-nos afirmar que o lado biológico, que trata da saúde, da orientação sexual e do conhecimento sobre o corpo (temas transversais e eixo temático dos PCN), e pautado como competência de desenvolvimento nas aulas de Educação Física. Esse bloco fornece ao aluno informações básicas sobre o próprio corpo, sua estrutura física e a interação com o meio social em que vive.

Portanto, saúde e qualidade de vida são temas, na teoria, bem preconizados de acordo com os PCN e Leis de Diretrizes e Bases da Educação Nacional - LDBEN. Mas qual o motivo de um assunto de suma importância não ter a ênfase necessária dentro das escolas no Ensino Médio?

\section{OBJETIVOS}

Não cabe ao estudo solucionar o sistema de ensino-aprendizagem nas escolas, todavia para desenvolver o tema proposto, necessitamos explicitar a verdadeira realidade no interior das escolas de Ensino Médio, para que possamos no final levantar propostas efetivas de ação no âmbito da qualidade de vida, saúde e atividade física na escola.

Desta forma, este estudo justifica-se em sistematizar ferramentas que possam trazer informações relativas as condições de conhecimento que os alunos possuem sobre a temática atividade física, saúde e qualidade de vida. Apos esse primeiro momento de compreensão da realidade da Escola, buscaremos realizar analises para aquisição de dados sobre os níveis individuais de saúde e aptidão física destes alunos, para que possamos aferir relações com enfoque a saúde. O levantamento de todos esses dados permitira gerar uma ampla discussão sobre conhecimento apreendido, conhecimento utilizado e o reflexo da tomada (ou não) de consciência sobre a importância da manutenção de um bem estar físico pautado nas questões da qualidade de vida. 


\section{METODOLOGIA}

\section{Abordagem e Tipo da Pesquisa}

Para fornecermos fundamentação teórica a discussão da monografia "As Aulas De Educação Física No Ensino Médio: Implicações nas Discussões Sobre as Temáticas Aprendizagem E Qualidade De Vida ", realizamos, num primeiro momento, uma pesquisa do tipo bibliográfica, pois esta nos deu oportunidade de adquirir amplas informações qualitativas relativas as diferentes temáticas da pesquisa.

Apos o contato com a literatura especifica do estudo de nossa temática, desenvolvemos uma pesquisa de campo, de característica transversal e quantitativa, porem os dados levantados serão tratados numa perspectiva qualitativa, nos quais buscaremos extrair o maior numero de informações sobre o binômio saúde e atividade física.

\section{Sujeitos}

Participaram do estudo 35 alunos (15 alunas e 20 alunos) em fase do Ensino Médio de uma Escola particular de Campinas. As alunas tinham idade media de 16,2 \pm 0,86 anos enquanto aos alunos 16,65 \pm 0,87 anos. Os alunos receberam um documento com explicações sobre a importância da pesquisa e todos trouxeram autorização do responsável para participação da mesma (anexos).

\section{Análise do Índice de Massa Corporal e do Fator de Risco Corbin}

Para a análise do Índice de Massa Corporal (IMC), medimos o peso e altura dos alunos em uma Balança Mecânica de Plataforma Welmy 104A. O calculo do IMC e predito pela equação: IMC = peso/altura ${ }^{2}$. Também analisamos o IMC do pai e mãe dos alunos, os quais enviaram tais dados por meio de uma questão especifica de nosso questionário. De acordo com a Organização Mundial da Saúde, ha 5 categorias e índices de classificação do IMC:

Abaixo do peso, IMC abaixo de 18,5; peso normal, IMC entre 18,5 e 24,9; sobrepeso, IMC entre 25 e 29,9; obesidade grau I, IMC entre 30 e 34,9; obesidade grau II, IMC entre 35 e 39,9 e obesidade grau III, IMC de 40 ou acima. Onde o peso saudável equivale ao peso normal.

\section{Analise do Conhecimento e Interesse em Temáticas Relativas Sobre Atividade Física e Saúde}

$\mathrm{O}$ comum em pesquisas que visam relacionar a temática atividade física e qualidade de vida e a utilização de questionários validados pela comunidade cientifica, tal como o World Health Organization Quality of Life (THE WHOQOL GROUP, 1994) e do Questionário International de Atividade Física 
(IPAQ). Porem, a especificidade destes questionários, em alguns casos de pesquisas, não permite a compreensão e delineamento de muitos fatores considerados importantes pelo pesquisador. Neste caso, ha a possibilidade de introduzir questões abertas, com o intuito de se fazer uma analise do discurso. Os dados obtidos foram analisados descritivamente por meio da Técnica de Analise de Conteúdo que tratou de descrever os conteúdos conforme a forma e o fundo. A analise da forma preocupa-se em estudar símbolos empregados, isto e, palavras ou temas. A analise de fundo centra seu interesse na investigação das referencias dos símbolos e pode revelar tendências verificadas no conteúdo da comunicação e, portanto, prevê a sistematização e a inferência, conforme defende Richardson (1989). Bardin (1979) conceitua a Analise de Conteúdo como técnica destinada a analise que visa subtrair das comunicações, indicadores (qualitativos ou não) que possibilitem fazer inferências de conhecimentos relativos as condições que permearam a emissão-recepção da mensagem comunicada. As questões buscaram contemplar a temática e os elementos importantes a ela subjacentes.

\section{RESULTADOS}

Os alunos delimitam como conceitos pertinentes a temática da atividade física e saúde três grandes micleos: componentes de aptidão física e preparação ao exercício físico, componentes esportivos e componentes preventivos. Dentro do micleo da aptidão física e preparação ao exercício físico, detectamos que ha $24,1 \%$ das respostas (alongamento, aquecimento, respiração, reflexos, controle da freqüência cardíaca, habilidades motoras [6,5\%-não consta na legenda] e aspectos psicológicos [1,6\%não consta na legenda]); no micleo dos componentes esportivos observamos 44\% das respostas (exercícios de forca, exercícios de velocidade, atividades de corrida e atividades esportivas e trabalho em equipe [4,8\%-não consta na legenda]), enquanto que no micleo dos componentes preventivos ha 31,9\% das respostas (condicionamento físico, alimentação, prevenção de doenças, benefícios da atividade física, limites do corpo e postura).

Dentro do universo dos conceitos desejados em atividade física e saúde pelos alunos que poderiam ser desenvolvidos nas aulas de Educação Física, ha um ponto que se sobressai aos demais, que e a falta de interesse em se aprofundar na temática (32\%). Observamos que os demais conceitos de interesse encontram-se diluídos, sobressaindo-se mais o interesse pela musculação (11\%).

45\% dos parentes do núcleo familiar dos alunos apresentam um quadro de doença cronicodegenerativa. Interessante também ressaltar que não houve nenhuma declaração, por parte dos alunos, a 
constatação de doença cronico-degenerativa, o que nos leva a refletir sobre os fatores ligados a herança genética familiar na influencia da instalação de uma patologia. Observamos a indicação de três das mais importantes causas de morte em virtude de doenças cronico-degenerativas: diabetes (41\%), hipertensão arterial (31\%) e doenças cardíacas (21\%), totalizando 93\% das patologias estabelecidas entre os parentes dos alunos.

Apesar dos alunos não apresentarem desenvolvimento de soprepeso/obesidade, as analises familiares mostraram que $72 \%$ dos pais e $47 \%$ das mães encontravam-se em condições de alerta, de acordo com o IMC e o fator de risco de Corbin ( $<<0.001$ quando comparados aos filhos).

\section{CONCLUSÃO}

Através de conceitos relevantes sobre atividade física, seus benefícios e sobre seus malefícios quando não orientada e/ou híper estimulada, com o foco no culto ao corpo, o qual a mídia vem fazendo com muita destreza, e sobre conhecimentos gerais de funcionamento corporal, durante e claro as aulas de EDF, as metas descritas acima poderão ser alcançadas.

Portanto, através deste estudo, iniciativas no âmbito de fornecer tal conhecimento para os alunos, em primeiro lugar os que nos auxiliaram como voluntários para a pesquisa, serão realizadas como estopim para alcançarmos, futuramente, o objetivo maior, que e inserir de vez assuntos tão pertinentes ao contexto moderno e atual de nossa sociedade na educação formal de nossos alunos, para que estes saiam formados com todo conhecimento sobre lingǘstica, ética, conhecimentos matemáticos $\mathrm{e}$ CONHECIMENTOS SOBRE SEU PROPRIO CORPO, para participação efetiva na sociedade.

\section{REFERÊNCIAS}

BENTO, J. O. As funções da educação fisicica. Revista Horizonte, n. 45, p. 135-146, 1991.

BOUCHARD, C. Can obesity be prevented? Nutrition Reviews, v. 54, p. S125-S130, 1996.

FORATTINI, O. P. Qualidade de vida e meio urbano: a cidade de São Paulo, Brasil. novos aspectos da saúde pública. Revista de Saúde Pública, v. 25, n. 02, p. 75-86, 1991.

MARCONI, M. A., LAKATOS, E. M. Técnicas de pesquisa. São Paulo: Atlas, 1986.

MARTIM, A. J., STOCKLER, M. Quality of life assessment health come research and practice. Evolution \& Health Professions, v. 21, p.141-156, 1998. 
MATTOS, M. G., NEIRA, M. G. Educação Física na adolescência: construindo o conhecimento na escola. Phorte, 2000.

McARDLE, W. D., KATCH, F. I., KATCH, V. L. Fisiologia do exercício: energia, nutrição e desempenho, 2003.

POWERS, S. K.; HOWLEY, E. T. Fisiologia do exercício: teoria e aplicação ao condicionamento e ao desempenho. São Paulo: Manole, 2000.

WORLD HEALTH ORGANIZATION. (WHO) Obesity: Preventing and Managing the global epidemic. W H O, 1997.

WHOQOL GROUP. The World Health Organization Quality of Life Assessment (WHOQOL): position paper from the The World Health Organization. Social Science and Medicine, v. 41, n. 10, p. 1403-1409, 1995. 


\section{Rodrigo Castelo Branco de Andrade}

Faculdades Integradas Metropolitana de Campinas

\section{Joaquim Antunes Neto}

Faculdades Integradas Metropolitana de Campinas

\section{Referência do artigo:}

\section{ABNT}

ANDRADE, R. C. B., NETO, J. A. As aulas de educação física no ensino médio: implicações nas discussões sobre as temáticas aprendizagem e qualidade de vida. Conexões, v. 6, p. 389-396, 2008.

\section{APA}

Andrade, R. C. B., \& Neto, J. A. (2008). As aulas de educação física no ensino médio: implicações nas discussões sobre as temáticas aprendizagem e qualidade de vida. Conexões, 6, 389-396.

\section{VANCOUVER}

Andrade RCB, Neto JA. As aulas de educação física no ensino médio: implicações nas discussões sobre as temáticas aprendizagem e qualidade de vida. Conexões, 2008; 6: 389-396. 